PROCEEDINGS OF THE

AMERICAN MATHEMATICAL SOCIETY

Volume 133, Number 10, Pages 2985-2995

S 0002-9939(05)07837-8

Article electronically published on March 22, 2005

\title{
A CHARACTERIZATION OF POSITIVE SELF-ADJOINT EXTENSIONS AND ITS APPLICATION TO ORDINARY DIFFERENTIAL OPERATORS
}

\author{
GUANGSHENG WEI AND YAOLIN JIANG
}

(Communicated by Joseph A. Ball)

\begin{abstract}
A new characterization of the positive self-adjoint extensions of symmetric operators, $T_{0}$, is presented, which is based on the Friedrichs extension of $T_{0}$, a direct sum decomposition of domain of the adjoint $T_{0}^{*}$ and the boundary mapping of $T_{0}^{*}$. In applying this result to ordinary differential equations, we characterize all positive self-adjoint extensions of symmetric regular differential operators of order $2 n$ in terms of boundary conditions.
\end{abstract}

\section{INTRODUCTION}

A linear operator $T$ with domain $D(T)$ in a Hilbert space $H$ is said to be a positive self-adjoint operator if $T=T^{*}$ and

$$
\lambda_{0}(T):=\inf \{(T u, u): u \in D(T),\|u\|=1\} \geq 0,
$$

where $T^{*}$ denotes the adjoint of $T$ and the constant $\lambda_{0}(T)$ is called the lower bound of $T$. It is well known [17, p. 115] that if a symmetric operator $T_{0}$ satisfies $\lambda_{0}\left(T_{0}\right) \geq 0$, then $T_{0}$ can be extended to a positive self-adjoint operator which is called a positive self-adjoint extension of $T_{0}$.

During the past several decades, there have been many works dealing with the problems associated with positive self-adjoint extensions. For any symmetric operator $T_{0}$ which is bounded below, K. Friedrichs [5] in 1934 constructed a boundpreserving self-adjoint extension $T_{F}$ by means of completing the inner product $\left(T_{0}, \cdot\right)$. This has come to be known as the Friedrichs extension and is a seminal result in analysis. The Friedrichs extension has been studied and applied by a great number of authors in the context of various differential operators; see, for example, [8, 9, 11] and the references therein. Furthermore, for any fixed real number $\mu_{0}$ which satisfies $\mu_{0} \leq \lambda_{0}\left(T_{0}\right)$, M. G. Krein [6] 7] in 1947 constructed all self-adjoint extensions $T$ such that $\lambda_{0}(T) \geq \mu_{0}$, for which the method used by Krein is similar to the von Neumann theory on self-adjoint extensions of symmetric operators in the "real" case. These extensions $T$ in our opinion may be called the bound-preserving

Received by the editors October 30, 2003 and, in revised form, May 17, 2004.

2000 Mathematics Subject Classification. Primary 47A20; Secondary 47E05, 34L05.

Key words and phrases. Friedrichs extension, positive self-adjoint extension, boundary condition.

This research was supported by the National Natural Science Foundation of P. R. China (No. 10071048). 
self-adjoint extensions. The Krein extension theory also has been studied and applied by a great number of authors; see, for example, [1]-[3] and the references therein. However, much less is known in applications of the abstract extension theory of Krein to ordinary differential operators, partly because the Krein theory is constructive and in practice, it is not easy to produce concrete realizations of boundary conditions of differential operators. Thus, with a view to applications to ordinary differential operators, it seems to be necessary to form a new approach to characterize the bound-preserving self-adjoint extensions, which is the motivation of the paper. Note that when $\lambda_{0}\left(T_{0}\right) \geq 0$ and $\mu_{0}=0$, the bound-preserving selfadjoint extensions reduce to the positive self-adjoint extensions of $T_{0}$. Conversely, once the positive self-adjoint extensions are known, the realizations of the boundpreserving self-adjoint extensions is then a simple matter to consider the operator $\hat{T}_{0}:=T_{0}-\mu_{0} I$ instead of $T_{0}$, where $I$ is the identity operator on $H$.

In this paper, we attempt to provide a new characterization for the positive self-adjoint extensions of symmetric operators in the case of finite deficiency indices. Note that, in order to obtain the left-definiteness of the Sturm-Liouville (SL) problems and the self-adjoint boundary conditions for the SL problems which have the same lowest eigenvalues, recently, the authors [13, 14] characterized all positive self-adjoint extensions of both regular and singular SL differential operators. This description is based on the Friedrichs extension and a direct sum decomposition of domain of the maximal SL differential operator. The purpose of the paper is to generalize this to abstract operators and form a general approach to describe the positive self-adjoint extensions. The proof is by means of the boundary mapping of the adjoint $T_{0}^{*}$ of the symmetric operator $T_{0}$, which was introduced in [12, 16] for characterizing the self-adjoint extensions of symmetric operators. This new characterization can be conventionally applied to ordinary differential operators. In the present paper, all positive self-adjoint extensions of symmetric regular differential operators of order $2 n$ are described in terms of boundary conditions.

This paper is organized as follows. Section 2 contains the main result characterizing all positive self-adjoint extensions of symmetric operators. The positive self-adjoint boundary conditions of differential operators are presented in Section 3.

Although we have only considered the case of finite deficiency indices in this paper, a similar result may be given in the case of countable infinite deficiency indices when combined with the works of [4] and [15].

\section{Positive SelF-ADjoint EXtensions}

Let $\mathbf{R}$ be the real line, let $\mathbf{C}$ be the complex field and let $\mathbf{C}^{m}=\left\{\alpha=\left(c_{1}, \ldots, c_{m}\right)\right.$ : $\left.c_{i} \in \mathbf{C}, i=1, \ldots, m\right\}$. We write a matrix $A$ with $m$ rows and $n$ columns as $A=$ $\left(a_{i j}\right)_{m \times n}$ or $A=\left(a_{i j}\right)_{1 \leq i \leq m, 1 \leq j \leq n}$, where $a_{i j}$ is the element of $A$ appearing in the $i^{\text {th }}$ row and $j^{\text {th }}$ column. In the case when $m=n$, we simply write $A=\left(a_{i j}\right)_{n}$ or $A=\left(a_{i j}\right)_{1 \leq i, j \leq n}$. If all elements of $A$ are zeros, we write $A$ as $0_{m \times n}$. Let $A^{T}$ and $A^{*}$ denote the transpose and Hermite adjoint of $A$, respectively.

Throughout this section let $H$ denote a Hilbert space over the complex field $\mathbf{C}$ with inner product $(\cdot, \cdot)$ and norm $\|\cdot\|$. Suppose that $T_{0}: D\left(T_{0}\right) \subset H \rightarrow H$ is an unbounded closed symmetric operator and $T_{0}^{*}$ denotes its adjoint. In what follows, when $\lambda_{0}\left(T_{0}\right)>0$, we will present a new characterization of the positive self-adjoint extensions of $T_{0}$, which is based on the Friedrichs extension of $T_{0}$, a direct sum 
decomposition of the domain of the adjoint $T_{0}^{*}$ and the boundary mapping of $T_{0}^{*}$. Now we give a detailed discussion for these.

It is well known [17] p. 123] that If $T_{0}$ is a closed symmetric operator with $\lambda_{0}\left(T_{0}\right)>-\infty$, then $T_{0} \subset T_{0}^{*}$ and the deficiency indices of $T_{0}$ are equal, which can be denoted by $m_{+}=m_{-}=: m$ (say, $\operatorname{def}\left(T_{0}\right)=m$ ), where

$$
m_{ \pm}:=\operatorname{dim}\left(N\left(T_{0}^{*} \mp i I\right)\right) \text {. }
$$

Here $N\left(T_{0}^{*}-i I\right)$ denotes the null space of the operator $T_{0}^{*}-i I$. Note that in the case of $\lambda_{0}\left(T_{0}\right)>-\infty, \operatorname{dim}\left(N\left(T_{0}^{*}-\lambda I\right)\right)=m$ as $\lambda \in \mathbf{C} \backslash\left[\lambda_{0}\left(T_{0}\right), \infty\right)$; see [17, Theorem 5.32]. In the following we always assume $m<\infty$.

Definition $2.1([5])$. Let $T_{0}$ be a symmetric operator which is bounded below. The operator $T_{F}$ is called the Friedrichs extension of $T_{0}$ if its domain $D\left(T_{F}\right)$ consists of all $y$ in $D\left(T_{0}^{*}\right)$ such that there exists a sequence $y_{k}$ in $D\left(T_{0}\right)$ satisfying

(i) $y_{k} \rightarrow y$ in $H$ as $k \rightarrow \infty$,

(ii) $\left(T_{0}\left(y_{k}-y_{n}\right), y_{k}-y_{n}\right) \rightarrow 0$ as $k, n \rightarrow \infty$, and the operator $T_{F}$ is the restriction of $T_{0}^{*}$ to $D\left(T_{F}\right)$.

As is well known [17] Theorem 5.38], if $T_{0}$ is bounded below, then its Friedrichs extension always exists and is a self-adjoint extension of $T_{0}$, which preserves the lower bound of $T_{0}$, i.e.,

$$
\lambda_{0}\left(T_{F}\right)=\lambda_{0}\left(T_{0}\right) .
$$

Lemma 2.2. Let $\lambda_{0}\left(T_{0}\right)>0$, let $\operatorname{def}\left(T_{0}\right)=m<\infty$ and let $T_{F}$ be the Friedrichs extension of $T_{0}$. Then

$$
D\left(T_{0}^{*}\right)=D\left(T_{F}\right) \dot{+} N\left(T_{0}^{*}\right),
$$

where the symbol $\dot{+}$ indicates that the sum is direct.

Proof. Equation (2.2) follows from the fact that $\lambda_{0}\left(T_{F}\right)>0$ and thus $D\left(T_{F}\right) \cap$ $N\left(T_{0}^{*}\right)=\{0\}$ and the fact that $D\left(T_{F}\right)$ has defect index $m$ in $D\left(T_{0}^{*}\right)$.

Since $T_{F}$ is a bound-preserving self-adjoint extension of $T_{0}$, if $\lambda_{0}\left(T_{0}\right)>0$, then the symmetric sesquilinear form $\left(T_{F} \cdot, \cdot\right)$ results in a positive definite inner product on the linear manifold $D\left(T_{F}\right)$. Denote its completion space by

$$
H_{F}:=\left(H_{F},(\cdot, \cdot)_{D}\right) .
$$

By the way, from Definition 2.1, we easily see that the linear manifold $D\left(T_{0}\right)$ is densely defined in $H_{F}$ with respect to this inner product $(\cdot, \cdot)_{D}$. Furthermore, by Lemma 2.2 , each $u \in D\left(T_{0}^{*}\right)$ can be uniquely represented as

$$
u=u_{F}+\sum_{i=1}^{m} c_{i} \theta_{i}, \quad u_{F} \in D\left(T_{F}\right), \quad \operatorname{span}\left\{\theta_{1}, \cdots, \theta_{m}\right\}=N\left(T_{0}^{*}\right) .
$$

Because of this, we will denote the above inner product henceforth by $(u, v)_{D}^{F}$ for any $u, v \in D\left(T_{0}^{*}\right)$, that is,

$$
(u, v)_{D}^{F}=\left(T_{F} u_{F}, v_{F}\right), \quad u, v \in D\left(T_{0}^{*}\right) .
$$

Definition 2.3. Let $T_{0}$ be a closed symmetric operator with equal deficiency indices $\operatorname{def}\left(T_{0}\right)=m<\infty$. If the linear mapping $\Gamma(\cdot): D\left(T_{0}^{*}\right) \mapsto \mathbf{C}^{2 m}$ is surjective and all $u_{0}$ in $D\left(T_{0}\right)$ satisfy $\Gamma\left(u_{0}\right)=0$, then $\Gamma(u)$ is called the boundary vector of $u$ in $D\left(T_{0}^{*}\right)$ and $\Gamma(\cdot)$ the boundary mapping of $T_{0}^{*}$. 
Similar to ordinary differential operators (see, for example, [9, pp. 51-52]), let $k$ be an integer with $0 \leq k \leq 2 m$ and $M$ be a $k \times 2 m$ matrix defined on $\mathbf{C}$ with rank $M=k$. For any such $M$ and a boundary mapping $\Gamma(\cdot)$ we define an operator $T(M)$ from $H$ into itself by

$$
\begin{aligned}
D(T(M)) & =\left\{u \in D\left(T_{0}^{*}\right): M \Gamma^{*}(u)=0\right\}, \\
T(M) u & =T_{0}^{*} u \quad(u \in D(T(M))) .
\end{aligned}
$$

When $k=0$ we have $M=0$ and $T(M)=T_{0}^{*}$, and when $k=2 m$ we have $T(M)=$ $T_{0}$. Here the matrix $M$ and $M \Gamma^{*}(u)=0$ may be called a boundary matrix and a boundary condition, respectively.

From (2.6) it is clear that $D(T(M))$ is a linear submanifold of $D\left(T_{0}^{*}\right)$ and satisfies $T_{0} \subseteq T(M) \subseteq T_{0}^{*}$ for any boundary matrix $M$. It was proved [16. Lemma 4] that if we choose appropriate boundary matrices, then all self-adjoint extensions of $T_{0}$ can be described in terms of (2.6). Note that all positive self-adjoint extensions (if any) are contained in the self-adjoint extensions. With this view the problem of describing positive self-adjoint extensions of $T_{0}$ now reduces to finding the self-adjoint boundary matrices $M$ in (2.6) such that $(T(M) u, u) \geq 0$ for all $u \in D(T(M))$.

Proposition 2.4. Let $T_{0}$ be a closed symmetric operator with $\lambda_{0}\left(T_{0}\right)>0$ and $\operatorname{def}\left(T_{0}\right)=m<\infty$. If both $\Gamma_{1}$ and $\Gamma_{2}$ are the boundary mappings of $T_{0}^{*}$, then there exists a $2 m \times 2 m$ nonsingular matrix $\Delta$ such that

$$
\Gamma_{1}(u)=\Gamma_{2}(u) \Delta, \quad \text { for all } u \in D\left(T_{0}^{*}\right) .
$$

Proof. (2.7) is immediately clear from Definition 2.3.

Proposition 2.5. Under the assumptions that $T_{0}$ is a closed symmetric operator with $\lambda_{0}\left(T_{0}\right)>0$ and $\operatorname{def}\left(T_{0}\right)=m<\infty$, and $\Gamma$ is a boundary mapping of $T_{0}^{*}$, then there exist two $2 m \times 2 m$ Hermitian matrices $A$ and $B$ such that for any $u \in D\left(T_{0}^{*}\right)$ the following identities hold:

$$
\begin{aligned}
& 2 \operatorname{Im}\left(T_{0}^{*} u, u\right)=\Gamma(u) A \Gamma^{*}(u), \\
& 2 \operatorname{Re}\left(T_{0}^{*} u, u\right)=2(u, u)_{D}^{F}+\Gamma(u) B \Gamma^{*}(u) .
\end{aligned}
$$

Here both matrices $A$ and $B$ are nonsingular and have zero signature.

Proof. Since $T_{F}$ is a self-adjoint extension of $T_{0}$ and $\operatorname{def}\left(T_{0}\right)=m$, it follows from [17. p. 239] that $T_{F}$ is an $m$-dimensional extension of $T_{0}$, and there exist $\theta_{m+i}$ in the quotient space $D\left(T_{F}\right) / D\left(T_{0}\right), 1 \leq i \leq m$, such that

$$
D\left(T_{F}\right)=D\left(T_{0}\right) \dot{+} \operatorname{span}\left\{\theta_{m+1}, \cdots, \theta_{2 m}\right\} .
$$

Since $\lambda_{0}\left(T_{0}\right)>0$, it follows that $\operatorname{dim}\left(N\left(T_{0}^{*}\right)\right)=m$. Let $N\left(T_{0}^{*}\right)=\operatorname{span}\left\{\theta_{1}, \ldots, \theta_{m}\right\}$. Then, for any $u \in D\left(T_{0}^{*}\right)$, we have

$$
u=u_{F}+\sum_{i=1}^{m} c_{i} \theta_{i} \quad \text { and } \quad u_{F}=u_{0}+\sum_{i=1}^{m} c_{m+i} \theta_{m+i}
$$


where $u_{F} \in D\left(T_{F}\right), u_{0} \in D\left(T_{0}\right)$. By (2.10) and (2.5), for any $u$ in $D\left(T_{0}^{*}\right)$, we have that $u=u_{0}+\sum_{i=1}^{2 m} c_{i} \theta_{i}, c_{i} \in \mathbf{C}, 1 \leq i \leq 2 m$, and

$$
\begin{aligned}
\left(T_{0}^{*} u, u\right) & =\left(T_{0}^{*}\left(u_{F}+\sum_{i=1}^{m} c_{i} \theta_{i}\right), u_{F}+\sum_{j=1}^{m} c_{j} \theta_{j}\right) \\
& =\left(T_{0}^{*} u_{F}, u_{F}\right)+\left(T_{0}^{*}\left(u_{0}+\sum_{i=m+1}^{2 m} c_{i} \theta_{i}\right), \sum_{j=1}^{m} c_{j} \theta_{j}\right) \\
& =(u, u)_{D}^{F}+\left(T_{0} u_{0}, \sum_{j=1}^{m} c_{j} \theta_{j}\right)+\sum_{i=m+1}^{2 m} \sum_{j=1}^{m} c_{i} \bar{c}_{j}\left(T_{0}^{*} \theta_{i}, \theta_{j}\right) \\
& =(u, u)_{D}^{F}+\sum_{i=m+1}^{2 m} \sum_{j=1}^{m} c_{i} \bar{c}_{j}\left(T_{0}^{*} \theta_{i}, \theta_{j}\right) .
\end{aligned}
$$

Thus, if we write $\Gamma_{1}(u)=\left(c_{1}, \ldots, c_{2 m}\right)$, then (2.11) implies

$$
\begin{aligned}
& 2 \operatorname{Im}\left(T_{0}^{*} u, u\right)=-i \Gamma_{1}(u) \hat{B}_{0} \Gamma_{1}^{*}(u), \\
& 2 \operatorname{Re}\left(T_{0}^{*} u, u\right)=2(u, u)_{D}^{F}+\Gamma_{1}(u) B_{0} \Gamma_{1}^{*}(u),
\end{aligned}
$$

where

$$
\hat{B}_{0}=\left(\begin{array}{cc}
0 & -B_{00}^{*} \\
B_{00} & 0
\end{array}\right) \quad \text { and } \quad B_{0}=\left(\begin{array}{cc}
0 & B_{00}^{*} \\
B_{00} & 0
\end{array}\right)
$$

with $B_{00}=\left(\left(T_{0}^{*} \theta_{m+i}, \theta_{j}\right)\right)_{1 \leq i, j \leq m}$. Note that $\theta_{1}, \ldots, \theta_{2 m}$ are linearly independent relative to $D\left(T_{0}\right)$. It is not hard to see from (2.11) and Definition 2.3 that $\Gamma_{1}$ is a boundary mapping of $T_{0}^{*}$. From [16. Lemma 4] we have rank $\hat{B}_{0}=2 m$ and rank $B_{00}=m$. This therefore shows that rank $B_{0}=2 m$ and its signature is 0 . Furthermore, by Proposition 2.4, there exists a nonsingular $2 m \times 2 m$ matrix $\Delta$ such that

$$
\Gamma_{1}(u)=\Gamma(u) \Delta
$$

for all $u$ in $D\left(T_{0}^{*}\right)$. Substituting this into (2.12-2.13), we obtain that $A=-i \Delta \hat{B}_{0} \Delta^{*}$, $B=\Delta B_{0} \Delta^{*}$, and (2.8-2.9) hold, thus completing the proof.

We are now in a position to prove the main result of the section: to characterize all positive self-adjoint extensions of $T_{0}$ under the assumptions $\lambda_{0}\left(T_{0}\right)>0$ and $\operatorname{def}\left(T_{0}\right)=m<\infty$.

Theorem 2.6. Let $T_{0}$ be a closed symmetric operator with $\lambda_{0}\left(T_{0}\right)>0$ and $\operatorname{def}\left(T_{0}\right)$ $=m<\infty$, and let $\Gamma$ be a boundary mapping of $T_{0}^{*}$ such that (2.8) and (2.9) hold. Then an operator $T$ is a positive self-adjoint extension of $T_{0}$ if and only if there exists an $m \times 2 m$ matrix $M$ such that

$$
\operatorname{rank} M=m, \quad M A^{-1} M^{*}=0,
$$

$$
M B^{-1} M^{*} \text { is a negative definite or negative semidefinite matrix, }
$$

and $T u=T_{0}^{*} u, u \in D(T)$, where

$$
D(T)=\left\{u \in D\left(T_{0}^{*}\right): M \Gamma^{*}(u)=0\right\} .
$$


Proof. Let us suppose that the operator $T$ is a positive self-adjoint extension of $T_{0}$, that is, $T$ is self-adjoint and satisfies $(T u, u) \geq 0$ for all $u \in D(T)$. From the self-adjointness of $T$ and [16, Lemma 4], there exists an $m \times 2 m$ matrix $M$ such that (2.16) and (2.18) are satisfied. On the other hand, we can show that $(T u, u) \geq 0$ is equivalent to

$$
\Gamma(u) B \Gamma^{*}(u) \geq 0 \quad \text { for all } u \in D(T) .
$$

Obviously, from (2.1), (2.5) and (2.9) we only need to prove (2.19). If it is not true, then there is an element $u_{1}$ in $D(T)$ satisfying $\Gamma\left(u_{1}\right) B \Gamma^{*}\left(u_{1}\right)=:-2 \varepsilon_{1}<0$. From the definition of the Friedrichs extension (see Definition 2.1) and the representation (2.4) of $u_{1}\left(u_{1}=u_{1 F}+\sum_{i=1}^{m} c_{i} \theta_{i}, u_{1 F} \in D\left(T_{F}\right)\right)$, we conclude that $D\left(T_{0}\right)$ is densely defined in $D\left(T_{F}\right)$ with respect to the inner product $(\cdot, \cdot)_{D}$ and, for the positive number $\varepsilon_{1} / 2$, there exists an element $u_{0}$ in $D\left(T_{0}\right)$ such that $\left(u_{1 F}-u_{0}, u_{1 F}-u_{0}\right)_{D} \leq$ $\varepsilon_{1} / 2$. Since $D(T)$ is an extension manifold of $D\left(T_{0}\right), u_{1}-u_{0} \in D(T)$ and

$$
\begin{aligned}
0 \leq\left(T\left(u_{1}-u_{0}\right), u_{1}-u_{0}\right) & =\operatorname{Re}\left(T\left(u_{1}-u_{0}\right), u_{1}-u_{0}\right) \\
& =\left(u_{1 F}-u_{0}, u_{1 F}-u_{0}\right)_{D}+(1 / 2) \Gamma\left(u_{1}\right) B \Gamma^{*}\left(u_{1}\right) \\
& \leq-(1 / 2) \varepsilon_{1}<0 .
\end{aligned}
$$

This contradiction shows that (2.19) holds.

Furthermore, note that the mapping $\Gamma(\cdot): D\left(T_{0}^{*}\right) \rightarrow \mathbf{C}^{2 m}$ is linear and surjective. If we write $A^{-1} M^{*}$ as $\left(\alpha_{1}^{*}, \ldots, \alpha_{m}^{*}\right)$ where $\alpha_{1}, \ldots, \alpha_{m} \in \mathbf{C}^{2 m}$, then from (2.16) and (2.18) we easily see that

$$
D(T)=\left\{u \in D\left(T_{0}^{*}\right): \Gamma(u) \in \operatorname{span}\left\{\alpha_{1}, \ldots, \alpha_{m}\right\}\right\} .
$$

This, combined with (2.19), yields that

$$
\left(\begin{array}{c}
\alpha_{1} \\
\vdots \\
\alpha_{m}
\end{array}\right) B\left(\alpha_{1}^{*}, \ldots, \alpha_{m}^{*}\right)=M A^{-1^{*}} B A^{-1} M^{*}
$$

is a positive definite or positive semidefinite matrix. In addition, from (2.14) and (2.15) we obtain that $A=-i \Delta \hat{B}_{0} \Delta^{*}$ and

$$
\begin{aligned}
A^{-1^{*}} B A^{-1} & =\Delta^{-1^{*}} \hat{B}_{0}^{-1^{*}} \Delta^{-1}\left(\Delta B_{0} \Delta^{*}\right) \Delta^{-1^{*}} \hat{B}_{0}^{-1} \Delta^{-1} \\
& =\Delta^{-1^{*}} \hat{B}_{0}^{-1^{*}} B_{0} \hat{B}_{0}^{-1} \Delta^{-1} \\
& =-\Delta^{-1^{*}} B_{0}^{-1} \Delta^{-1} \\
& =-B^{-1}
\end{aligned}
$$

which shows that (2.17) holds. Thus, the necessary part of Theorem 2.6 is proved.

Conversely, if there is an $m \times 2 m$ matrix $M$ that satisfies (2.16), (2.17) and $D(T)$ satisfies (2.18), then, from [16. Lemma 4], we conclude that the operator $T$ is self-adjoint. If, in addition, we write $A^{-1} M^{*}=\left(\alpha_{1}^{*}, \ldots, \alpha_{m}^{*}\right)$, then by $(2.19),(2.21)$ and the above proof, we can conclude that $(T u, u) \geq 0$ for all $u \in D(T)$. This shows that $T$ is a positive self-adjoint extension of $T_{0}$. We complete the proof of Theorem 2.6. 


\section{Positive Self-Adjoint Extensions of DifFEREntial operators}

Let $l$ denote the formally symmetric differential expression defined by

$$
l y=\frac{1}{w} \sum_{i=0}^{n}(-1)^{i}\left(p_{n-i} y^{(i)}\right)^{(i)}, \quad t \in I:=[a, b] \subset \mathbf{R} .
$$

We assume that the coefficient functions $p_{i}, 0 \leq i \leq n$, and $w$ satisfy the following basic conditions:

$$
1 / p_{0}, p_{1}, \ldots, p_{n}, w \in L^{1}(I, \mathbf{R}), \quad w>0, p_{0}>0 \text { a.e. on } I
$$

where $L^{1}(I, \mathbf{R})$ denotes the set of Lebesgue integrable real functions on $I$. The basic conditions ensure that the expression $l$ is regular on $[a, b]$. Based on (3.2) we define the formal quasi-derivatives (up to order $2 n$ ) of a function $y$ to be the functions $y^{[0]}=y, y^{[1]}, \ldots, y^{[2 n]}$ given by

$$
y^{[k]}= \begin{cases}y^{(k)}, & 0 \leq k \leq n-1, \\ p_{0} y^{(n)}, & k=n \\ p_{k-n} y^{(2 n-k)}-\left\{y^{[k-1]}\right\}^{\prime}, & n+1 \leq k \leq 2 n\end{cases}
$$

where $y^{(k)}$ is the usual $k$ th derivative (see [10, Sect. 15.2]). The expression $l$ is then given by

$$
l y=w^{-1} y^{[2 n]} .
$$

The expression $l$ will be considered throughout the section in the weighted Hilbert space $L_{w}^{2}(I)$ of Lebesgue measurable functions which are square integrable with weight $w$ and with inner product and norm defined by $(f, g)=\int_{I} f(t) \overline{g(t)} w(t) d t$ and $\|f\|=(f, f)^{1 / 2}$. Associated with the expression $l$, three differential operators $L_{\max }, L_{\min }, L_{F}$, respectively called the maximal operator, minimal operator and Friedrichs extension of $L_{\mathrm{min}}$, are defined as follows (see [10, Section 17]): Let

$$
\begin{aligned}
D\left(L_{\max }\right) & =\left\{y \in L_{w}^{2}(I): y^{[k]} \in A C(I), 1 \leq k \leq 2 n-1, \text { and } l y \in L_{w}^{2}(I)\right\}, \\
D\left(L_{\min }\right) & =\left\{y \in D\left(L_{\max }\right): R_{2 n}(y)(a)=0=R_{2 n}(y)(b)\right\} \\
D\left(L_{F}\right) & =\left\{y \in D\left(L_{\max }\right): R_{n}(y)(a)=0=R_{n}(y)(b)\right\}
\end{aligned}
$$

where

$$
R_{k}(y)(t)=\left(y^{[0]}(t), y^{[1]}(t), \ldots, y^{[k-1]}(t)\right), \quad t \in[a, b]
$$

and $k$ is an integer with $1 \leq k \leq 2 n$. Then

$$
\begin{aligned}
L_{\max } y & =l y, & & y \in D\left(L_{\max }\right), \\
L_{\min } y & =l y, & & y \in D\left(L_{\min }\right), \\
L_{F} y & =l y, & & y \in D\left(L_{F}\right) .
\end{aligned}
$$

It is well known [10, Sect. 17] that $D\left(L_{\max }\right), D\left(L_{\min }\right)$ and $D\left(L_{F}\right)$ all are dense in $L_{w}^{2}(I)$ (therefore, $L_{\max }$ has a unique adjoint $\left.L_{\max }^{*}\right), L_{\min }^{*}=L_{\max }$, and $L_{\min }$ is a semi-bounded, closed, symmetric operator with deficiency indices $\operatorname{def}\left(L_{\min }\right)=2 n$ and lower bound $\lambda_{0}\left(L_{\min }\right)$. 
Denote

$$
\begin{gathered}
R(y)=\left(R_{2 n}(y)(a), R_{2 n}(y)(b)\right), \quad J_{n}=\left(\delta_{i,(n+1-j)}\right)_{1 \leq i, j \leq n}, \\
\hat{J}_{2 n}=\left(\begin{array}{cc}
0 & J_{n} \\
-J_{n} & 0
\end{array}\right), \quad \tilde{J}_{4 n}=\left(\begin{array}{cc}
-\hat{J}_{2 n} & 0 \\
0 & \hat{J}_{2 n}
\end{array}\right), \\
{[y, z](t)=R_{2 n}(y)(t) \hat{J}_{2 n} R_{2 n}^{*}(z)(t),}
\end{gathered}
$$

where $\delta_{i j}$ denotes the Kronecker delta function. For any $y, z \in D\left(L_{\max }\right)$ it is noted that the Green's formula ([10 p. 50]) now reads as

$$
\int_{a}^{b}[(l y) \bar{z}-y(\overline{l z})] w(t) d t=[y, z](b)-[y, z](a)=R(y) \tilde{J}_{4 n} R^{*}(y) .
$$

It is not hard to verify from Definition $2.3,(3.6)$ and $(3.10)$ that $R(\cdot)$ is a boundary mapping of $L_{\min }^{*}\left(=L_{\max }\right)$.

Let $\theta_{1}, \ldots, \theta_{2 n}$ denote the solutions of the equation $l y=0$, which satisfy the following initial value conditions:

$$
\left(R_{2 n}\left(\theta_{j}\right)(a)\right)_{1 \leq j \leq n}=\left(0_{n}, I_{n}\right)=\left(R_{2 n}\left(\theta_{n+j}\right)(b)\right)_{1 \leq j \leq n},
$$

where $I_{n}$ denotes the $n \times n$ identity matrix. For any $t \in[a, b]$, denote by

$$
\begin{array}{ll}
\Theta_{11}(t)=\left(\theta_{i}^{[j-1]}(t)\right)_{1 \leq i, j \leq n}, & \Theta_{12}(t)=\left(\theta_{i}^{[n-1+j]}(t)\right)_{1 \leq i, j \leq n}, \\
\Theta_{21}(t)=\left(\theta_{n+i}^{[j-1]}(t)\right)_{1 \leq i, j \leq n}, & \Theta_{22}(t)=\left(\theta_{n+i}^{[n-1+j]}(t)\right)_{1 \leq i, j \leq n} .
\end{array}
$$

Lemma 3.1. If $\lambda_{0}\left(L_{\min }\right)>0$, then $\theta_{1}, \ldots, \theta_{2 n}$ are linearly independent and

$$
\operatorname{rank} \Theta_{21}(a)=n=\operatorname{rank} \Theta_{11}(b), \quad \Theta_{11}(b)=-J_{n} \Theta_{21}^{*}(a) J_{n} .
$$

Proof. By the fact that $\lambda_{0}\left(L_{F}\right)=\lambda_{0}\left(L_{\min }\right)>0$, we easily see that 0 belongs to the resolvent set of $L_{F}$. Note that $\theta_{1}, \ldots, \theta_{n}$ and $\theta_{n+1}, \ldots, \theta_{2 n}$ are linearly independent respectively. If $\theta_{1}, \ldots, \theta_{2 n}$ are linearly dependent, then there exist constants $c_{i}$, $1 \leq i \leq 2 n$, such that $\sum_{i=1}^{n} c_{i} \theta_{i}=\sum_{i=1}^{n} c_{n+i} \theta_{n+i}=: \theta_{0} \not \equiv 0$ and $R_{n}\left(\theta_{0}\right)(a)=$ $0=R_{n}\left(\theta_{0}\right)(b)$, which shows that 0 is an eigenvalue of the Friedrichs extension $L_{F}$. This contradicts the prerequisite assumption and therefore $\theta_{1}, \ldots, \theta_{2 n}$ are linearly independent. If rank $\Theta_{11}(b)<n$, then there exist constants $c_{i}, 1 \leq i \leq n$, such that $\sum_{i=1}^{n} c_{i} \theta_{i}=: \theta_{0} \not \equiv 0$, which together with (3.11) and (3.7) also shows that 0 is an eigenvalue of $L_{F}$. Hence rank $\Theta_{11}(b)=n$. Furthermore, from the Green's formula (3.10), it is easily verified that $\left[\theta_{i}, \theta_{n+j}\right](a)=\left[\theta_{i}, \theta_{n+j}\right](b), 1 \leq i, j \leq n$. Thus, we obtain

$$
\left(\Theta_{11}(a), \Theta_{12}(a)\right) \hat{J}_{2 n}\left(\begin{array}{c}
\Theta_{21}^{*}(a) \\
\Theta_{22}^{*}(a)
\end{array}\right)=\left(\Theta_{11}(b), \Theta_{12}(b)\right) \hat{J}_{2 n}\left(\begin{array}{c}
\Theta_{21}^{*}(b) \\
\Theta_{22}^{*}(b)
\end{array}\right) .
$$

It follows from $(3.11)$ that $\left(\Theta_{11}(a), \Theta_{12}(a)\right)=\left(0_{n}, I_{n}\right)=\left(\Theta_{21}(b), \Theta_{22}(b)\right)$. Substituting this into (3.15), we obtain (3.14), thus completing the proof.

Let

$$
B=\left(\begin{array}{cccc}
0 & -J_{n} & 0 & 0 \\
-J_{n} & B_{22} & 0 & -2 J_{n} \Theta_{21}^{-1}(a) \\
0 & 0 & 0 & J_{n} \\
0 & -2 \Theta_{21}^{-1^{*}}(a) J_{n} & J_{n} & B_{44}
\end{array}\right)
$$


where

$$
\begin{aligned}
& B_{22}=-\Theta_{22}^{*}(a) \Theta_{21}^{-1^{*}}(a) J_{n}-J_{n} \Theta_{21}^{-1}(a) \Theta_{22}(a), \\
& B_{44}=\Theta_{12}^{*}(b) \Theta_{11}^{-1^{*}}(b) J_{n}+J_{n} \Theta_{11}^{-1}(b) \Theta_{12}(b) .
\end{aligned}
$$

Lemma 3.2. Let $\lambda_{0}\left(L_{\min }\right)>0$. Then for any $y \in D\left(L_{\max }\right)$ the following identities hold:

$$
\begin{aligned}
& 2 \operatorname{Im}\left(L_{\max } y, y\right)=-i R(y) \tilde{J}_{4 n} R^{*}(y), \\
& 2 \operatorname{Re}\left(L_{\max } y, y\right)=2(y, y)_{D}^{F}-R(y) B^{-1} R^{*}(y) .
\end{aligned}
$$

Proof. Clearly, (3.17) can be directly obtained from the Green's formula (3.10). For any $y \in D\left(L_{\max }\right)$, it follows from Lemma 2.2, (3.7) and (3.11) that $y=y_{F}+$ $\sum_{i=1}^{2 n} c_{i} \theta_{i}, y_{F} \in D\left(L_{F}\right)$, and

$$
\begin{aligned}
\left(L_{\max } y, y\right) & =\left(L_{\max } y_{F}, y_{F}+\sum_{i=1}^{2 n} c_{i} \theta_{i}\right) \\
& =\left(y_{F}, y_{F}\right)_{D}+\left.\left[y_{F}, \sum_{i=1}^{2 n} c_{i} \theta_{i}\right](t)\right|_{a} ^{b} \\
& =(y, y)_{D}^{F}+\left.\left(-R_{2, n}\left(y_{F}\right)(t) J_{n}, 0_{1 \times n}\right)\left(\begin{array}{cc}
\Theta_{11}^{*}(t) & \Theta_{21}^{*}(t) \\
\Theta_{12}^{*}(t) & \Theta_{22}^{*}(t)
\end{array}\right)\left(\begin{array}{c}
\alpha_{1}^{*}(y) \\
\alpha_{2}^{*}(y)
\end{array}\right)\right|_{a} ^{b} \\
& =(y, y)_{D}^{F}+R_{2, n}\left(y_{F}\right)(a) J_{n} \Theta_{21}^{*}(a) \alpha_{2}^{*}(y)-R_{2, n}\left(y_{F}\right)(b) J_{n} \Theta_{11}^{*}(b) \alpha_{1}^{*}(y),
\end{aligned}
$$

where

$$
\begin{gathered}
R_{2, n}(y)(t)=\left(y^{[n]}(t), \ldots, y^{[2 n-1]}(t)\right), \\
\alpha_{1}(y)=\left(c_{1}, \ldots, c_{n}\right) \quad \text { and } \quad \alpha_{2}(y)=\left(c_{n+1}, \ldots, c_{2 n}\right) .
\end{gathered}
$$

If we write

$$
\Gamma_{0}(y)=\left(R_{2, n}\left(y_{F}\right)(a), R_{2, n}\left(y_{F}\right)(b), \alpha_{1}(y), \alpha_{2}(y)\right),
$$

then $\Gamma_{0}(\cdot)$ is a boundary mapping of $L_{\max }\left(=L_{\min }^{*}\right)$ by Definition 2.3, and

$$
2 \operatorname{Re}\left(L_{\max } y, y\right)=2(y, y)_{D}^{F}-\Gamma_{0}(y) G \Gamma_{0}^{*}(y),
$$

where

$$
G=\left(\begin{array}{cccc}
0 & 0 & 0 & -J_{n} \Theta_{21}^{*}(a) \\
0 & 0 & J_{n} \Theta_{11}^{*}(b) & 0 \\
0 & \Theta_{11}(b) J_{n} & 0 & 0 \\
-\Theta_{21}(a) J_{n} & 0 & 0 & 0
\end{array}\right) .
$$

Furthermore, from (3.7) and (3.11) we have

$$
\begin{aligned}
& R_{2 n}(y)(a)=\left(0_{1 \times n}, R_{2, n}\left(y_{F}\right)(a)\right)+\left(\alpha_{1}(y), \alpha_{2}(y)\right)\left(\begin{array}{cc}
0 & I_{n} \\
\Theta_{21}(a) & \Theta_{22}(a)
\end{array}\right), \\
& R_{2 n}(y)(b)=\left(0_{1 \times n}, R_{2, n}\left(y_{F}\right)(b)\right)+\left(\alpha_{1}(y), \alpha_{2}(y)\right)\left(\begin{array}{cc}
\Theta_{11}(b) & \Theta_{12}(b) \\
0 & I_{n}
\end{array}\right) .
\end{aligned}
$$

This then implies $R(y)=\Gamma_{0}(y) \Delta$ with

$$
\Delta=\left(\begin{array}{cccc}
0 & I_{n} & 0 & 0 \\
0 & 0 & 0 & I_{n} \\
0 & I_{n} & \Theta_{11}(b) & \Theta_{12}(b) \\
\Theta_{21}(a) & \Theta_{22}(a) & 0 & I_{n}
\end{array}\right) .
$$


Simple calculations show that $B=\Delta^{*} G^{-1} \Delta, B^{-1}=\Delta^{-1} G \Delta^{-1^{*}}$ and (3.18) holds. This completes the proof.

In applying Theorem 2.6 to the minimal differential operator $L_{\min }$ we can directly obtain the positive self-adjoint extensions of $L_{\min }$ when $\lambda_{0}\left(L_{\min }\right)>0$. This result is stated in the following.

Theorem 3.3. Let the differential expression $l$ satisfy the basic conditions (3.2) and let $\lambda_{0}\left(L_{\min }\right)>0$. Then an operator $L$ is a positive self-adjoint extension of $L_{\min }$ if and only if there exists a $2 n \times 4 n$ matrix $M$ such that

$$
\text { rank } M=2 n, \quad M \tilde{J}_{4 n} M^{*}=0,
$$

$M B M^{*}$ is a positive definite or positive semidefinite matrix,

and $L y=L_{\max } y, y \in D(L)$, where

$$
D(L)=\left\{y \in D\left(L_{\max }\right): M R^{*}(y)=0\right\} .
$$

Here the boundary mapping $R(\cdot)$ and the matrix $B$ are defined as (3.9) and (3.16), respectively.

\section{REFERENCES}

1. A. Alonso and B. Simon, The Birman-Krein-Vishik theory of self-adjoint extensions of semibounded operators, J. Operator Theory 4 (1980), 251-270. MR0595414 (81m:47038)

2. Y. Arlinskii and E. Tsekanovskii, On von Neumann's problem in extension theory of nonnegative operators, Proc. Amer. Math. Soc. 131 (2003), 3143-3154. MR1992855 (2004h:47034)

3. M. S. Birman, On the self-adjoint extensions of positive definite operators, Mat. Sb. 38 (1956), 431-450. MR0080271 (18:220d)

4. W. N. Everitt and A. Zettl, Differential operators generated by a countable number of quasi-differential expressions on the real line, Proc. London Math. Soc. 64 (1992), 524-544. MR1152996 (93k:34182)

5. K. Friedrichs, Spektraltheorie halbbeschrankter operatoren, Math. Ann. 109 (1934), 465-487.

6. M. G. Krein, The theory of selfadjoint extensions of semibounded Hermitian transformations and its applications, I, Mat. Sbornik 20 (1947), 431-495 (in Russian). MR.0024574 (9:515c)

7. M. G. Krein, The theory of selfadjoint extensions of semibounded Hermitian transformations and its applications, II, Mat. Sbornik 21 (1947), 365-404 (in Russian). MR0024575 (9:515d)

8. M. Marletta and A. Zettl, The Friedrichs extension of singular differential operators, J. Differential Equations 160 (2000), 404-421. MR.1736997 (2000m:47058)

9. M. Möller and A. Zettl, Symmetric differential operators and their Friedrichs extension, J. Differential Equations 115 (1995), 50-69. MR1308604 (96a:34161)

10. N. A. Naimark, Linear Differential Operators, vol. II, Ungar, New York, 1968. MR0262880 (41:7485)

11. H.-D. Niessen and A. Zettl, Singular Sturm-Liouville problems: The Friedrichs extension and comparison of eigenvalues, Proc. London Math. Soc. 64 (1992), 545-578. MR 1152997 (93e:47060)

12. G. Wei, A new description of self-adjoint domains of symmetric operators, J. of Inner Monogolia University 27 (1996), 305-310. (in Chinese). MR.1440615 (98c:47031)

13. G. Wei and J. Wu, Characterization of left-definiteness of Sturm-Liouville problems, Math. Nachr., 2004 (accepted for publication).

14. G. Wei and Z. Xu, A characterization of boundary conditions for regular Sturm-Liouville problems which have the same lowest eigenvalues, Rocky Mountain J. Math. 2003 (accepted for publication).

15. G. Wei and Z. Xu, On self-adjoint extensions of symmetric differential operators with countably infinite deficiency indices, Advances in Math. 29 (2000), 227-234. (in Chinese). MR 1789424 (2001i:47071) 
16. G. Wei, Z. Xu and J. Sun, Self-adjoint domains of products of differential expressions, J. Differential Equations 174 (2001), 75-90. MR.1844524 (2002d:47063)

17. J. Weidmann, Linear Operators in Hilbert Spaces, Springer-Verlag, Berlin/New York, 1980. MR0566954 (81e:47001)

Research Center for Applied Mathematics and Institute for Information and System Science, Xi'An Jiaotong University, Xi'An 710049, People's Republic of China

E-mail address: weimath@pub.xaonline.com

E-mail address: isystem@vip.sina.com

Research Center for Applied Mathematics and Institute for Information and System Science, Xi'an Jiaotong University, Xi'an 710049, People's Republic of China

E-mail address: yljiang@xjtu.edu.cn 